\title{
Um laboratório portátil de baixo custo: medição de $g$ utilizando um pêndulo e a placa Raspberry $\mathrm{Pi}^{+*}$
}

\author{
Thiago Corrêa Almeida ${ }^{1}$ \\ Instituto de Aplicação Fernando Rodrigues da Silveira - CAp/UERJ \\ Eugênio de Carvalho Dias ${ }^{2}$ \\ Bolsista de Iniciação Científica - FAPERJ \\ Amanda da Silva Julião ${ }^{3}$ \\ Bolsista de Extensão - UERJ \\ Rio de Janeiro - RJ
}

\section{Resumo}

Neste artigo apresentaremos a placa Raspberry Pi como solução de baixo custo para um laboratório portátil com múltiplas aplicações, podendo ser utilizado tanto no ensino básico quanto no ensino superior. Utilizaremos a placa com um LDR (Light Dependent Resistor) para medir o período de um pêndulo e obter a aceleração da gravidade. Apesar de simples, o experimento possui excelente acordo com valor encontrado na literatura, diferindo em apenas $0,07 \%$.

Palavras-chave: Raspberry Pi; Pêndulo; Laboratório Portátil; Aceleração da Gravidade.

\begin{abstract}
In this article we will present the Raspberry Pi board as a low cost solution to a portable laboratory with multiple applications that can be used in basic education and in higher education. We will use the board with a
\end{abstract}

\footnotetext{
+A low cost portable laboratory: measuring $g$ with a pendulum and Raspberry P $i$

* Recebido: setembro de 2016.

Aceito: abril de 2017.

${ }^{1}$ E-mail: thiagoca3@yahoo.com.br

2 E-mail: eugenio_nintendo@hotmail.com

3 E-mail: amandajuliao.nave@gmail.com
} 
LDR (Light Dependent Resistor) for measuring the period of a pendulum and obtain the gravitational acceleration. Although simple, the experiment has excellent agreement with the value found in the literature, differing by only $0.07 \%$.

Keywords: Raspberry Pi; Pendulum; Portable Laboratory; Gravitational Acceleration.

\section{Introdução}

É inquestionável a importância do laboratório didático no processo de ensino e aprendizagem, tanto no ensino básico quanto no ensino superior. Algumas universidades contam com laboratórios equipados com kits da Pasco, da Phywe e da Cidepe, mas, infelizmente, nem todas possuem meios de adquiri-los, ou ainda, de repor componentes quando estes sofrem avarias ou se tornam obsoletos. No ensino básico a realidade é ainda mais difícil, poucas escolas contam com um laboratório didático minimamente equipado, inviabilizando ao professor associar a teoria com a prática. Neste sentido, a partir da década de 90 tivemos diversos trabalhos propondo a utilização do PC, através de sua porta paralela, para aquisição de dados, sendo utilizado como termômetro, gerador de onda quadrada (FAGUNDES, 1995), e outras aplicações (SOUSA, 1998). Na literatura recente podemos encontrar trabalhos que se utilizam de uma outra ferramenta muito valiosa, o microcontrolador Arduíno, que além do baixo custo possui uma grande comunidade de usuários com vasto material para consulta disponível, tornando sua utilização acessível para todos. Podemos encontrar sua utilização associado a sensores para diversos estudos, como a medição da aceleração da gravidade através da queda livre de um corpo (CORDOVA, 2016), estudo de oscilações e transferência de calor (SOUZA, 2011), estudo de carga e descarga em capacitor (CAVALCANTE, 2011), e outros.

No presente trabalho, apresentamos o Raspberry Pi (UPTON, 2013), um computador completo do tamanho de um smartphone que custa cerca de 35 US\$ e, quando associado a simples sensores, pode se tornar uma excelente opção para um laboratório portátil completo de baixo custo. A placa, lançada inicialmente em 2012 pela Raspberry Pi Foundation, já se encontra em sua terceira versão, que conta com um processador ARM de quatro núcleos (semelhante aos processadores de smartphone), memória RAM de $1 \mathrm{~GB}$ e armazenamento em cartão MicroSDHC. Seu consumo é baixo, apenas $800 \mathrm{~mA}$, e seu sistema operacional principal é o Raspbian, um sistema operacional de código aberto baseado em Debian. A placa Raspberry P $i$ (Fig. 1) tem vasta aplicação educacional no Reino Unido, e a Fundação tem investido em programas de treinamento para que professores possam utilizar a placa em suas escolas. Recentemente a Agência Espacial do Reino Unido promoveu uma competição chamada Astro Pi, onde alunos da escola primária e secundária elaboraram programas que realizaram experimentos e medidas diversas em placas Raspberry $P i$ a bordo da Estação Espacial Internacional, em missão liderada pelo astronauta Tim Peake. 
O que permite à placa esta gama de aplicações é seu conector GPIO (General purpose input-output), em destaque na Fig. 1, onde podem ser conectados diversos sensores de baixo custo para aquisição de dados, assim como no Arduíno. A vantagem do Raspberry Pi em relação ao Arduíno é que ele já é um computador completo, bastando conectá-lo a um monitor (ou TV, ou projetor) via HDMI ou vídeo composto, a um teclado e mouse para utilizá-lo, enquanto o Arduíno é apenas um microcontrolador, ou seja, para sua utilização é preciso adicionalmente um computador. Em salas de aula com data show, basta conectar o Raspberry Pi ao mesmo e realizar as experiências em tempo real com os alunos acompanhando os resultados na projeção.

Para demonstrar a utilização da placa realizamos um estudo com o pêndulo, utilizandoo para o cálculo da aceleração da gravidade. O Raspberry P $i$ é utilizado com um $L D R$ para obter o período do pêndulo de maneira indireta. O circuito utilizado é simples, assim como o programa escrito em linguagem python (MENEZES, 2014), disponível no apêndice A.

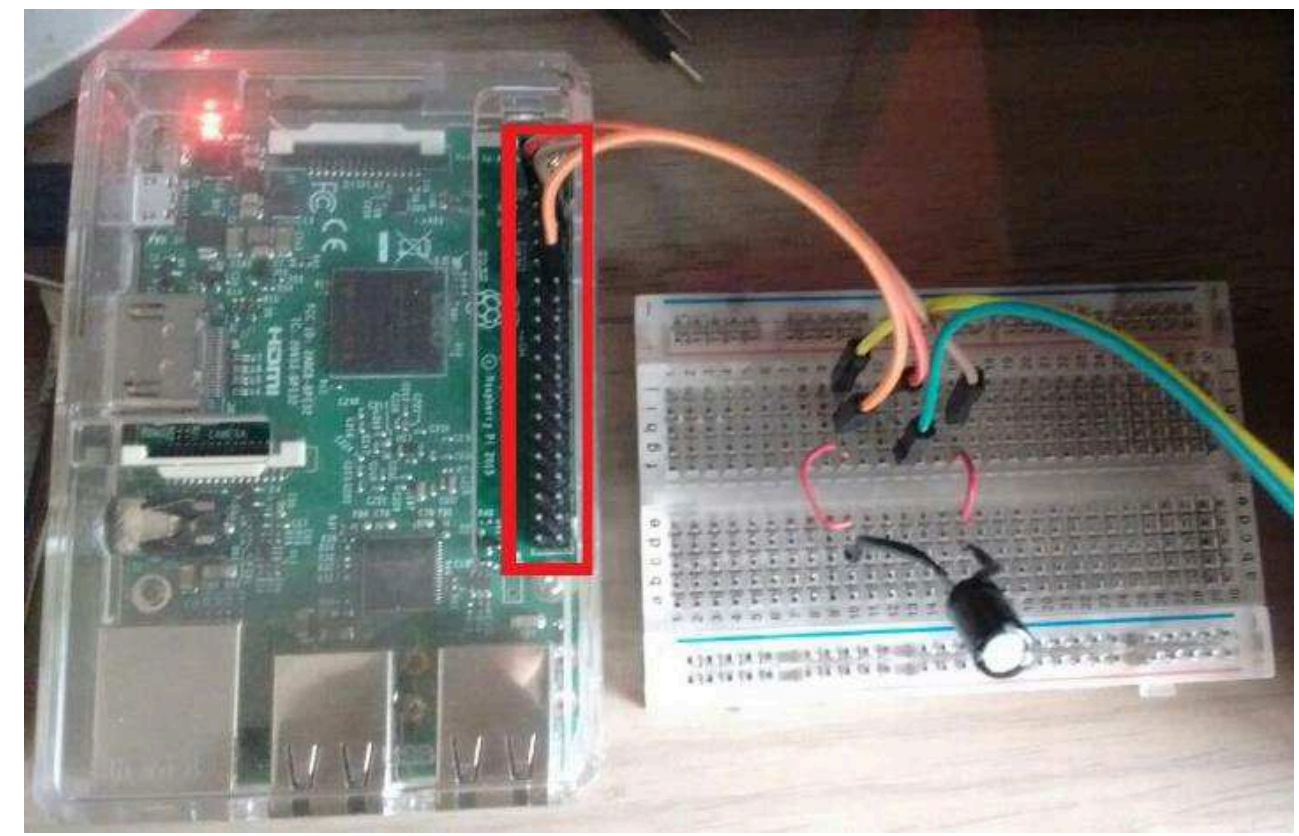

Fig. 1-Placa Raspberry Pi e o circuito utilizado. No destaque o conector GPIO.

Na literatura recente podemos encontrar diversas aplicações do Raspberry Pi, como sua utilização na elaboração de um sistema de baixo custo para análise de água (CHANDRAPPA, 2017), ferramenta acessível para modernização dos cursos de engenharia elétrica na África (MAINA, 2016), na construção de um sistema moderno de monitoramento de temperatura para laboratórios didáticos (NATARAJAN, 2017), e também como dispositivo de streaming em astronomia (MAULANA, 2016). 


\section{O pêndulo simples}

A análise do pêndulo simples pode ser encontrada em diversos livros didáticos no estudo do oscilador harmônico (NUSSENZVEIG, 2002). O período do pêndulo, na aproximação de pequenas oscilações, é dado por

$$
T_{0}=2 \pi \sqrt{\frac{L}{g}},
$$

no qual L é o comprimento do pêndulo e $g$ a aceleração da gravidade local. Uma equação exata para o pêndulo, em qualquer ângulo, pode ser escrita na forma

$$
T=\frac{2 \pi}{\operatorname{agm}(1, \cos (\theta / 2))} \sqrt{\frac{L}{g}},
$$

sendo $\operatorname{agm}(a, b)$ uma função conhecida como média aritmética-geométrica, e consiste em realizar iterações onde os novos passos são as médias geométrica e aritmética dos passos anteriores. As iterações são continuamente realizadas até que se obtenha convergência. Um estudo detalhado sobre esta solução exata para o pêndulo pode ser encontrado em Carvalhaes (2008). A definição de "pequenas oscilações", onde a Eq. (1) é válida, é um pouco obscura. Alguns autores consideram oscilações onde o erro introduzido pela aproximação seja inferior a $1 \%$, sendo assim podemos utilizar a Eq. (1) até um ângulo máximo de aproximadamente $23^{\circ}$ (AGGARWAL, 2005). Livros didáticos de nível superior afirmam apenas que a equação é válida para "pequenas oscilações", mas sem definir um limite. Nussenszveig (2002) atenta que para um ângulo de $10^{\circ}$ o erro relativo introduzido é de $0,5 \%$, já Halliday (2009) aponta que para um ângulo de $5^{\circ}$ o erro relativo é de $0,1 \%$, no entanto nenhum dos dois estipula $10^{\circ}$ ou $5^{\circ}$ como limite para as "pequenas oscilações” onde é válido utilizar a Eq. (1).

$\mathrm{Na}$ abordagem do pêndulo simples a resistência com o ar não é considerada e nem o atrito entre o fio e o suporte. Na análise de nosso pêndulo, utilizaremos a abordagem do pêndulo simples, pelo fato das forças dissipativas neste experimento não apresentarem influência considerável que inviabilize ou introduza um erro expressivo.

\section{Arranjo experimental}

O arranjo experimental é composto por duas partes, sendo a primeira o pêndulo e a segunda o instrumento de medição, que discutiremos em seguida. A placa Raspberry Pi utilizada foi a última lançada, versão 3, modelo B. No entanto, o experimento pode ser realizado perfeitamente com qualquer uma das versões anteriores. Apresentamos também uma seção com o passo a passo para configurar a placa e rodar o programa.

\section{III.1 Pêndulo}

A montagem do pêndulo foi realizada com utilização de materiais de simples aquisição, que podem ser encontrados facilmente em casa. São eles: um suporte (foi utilizado um 
suporte acrílico de um pêndulo decorativo), um palito de churrasco, fita adesiva, linha de costura, agulha, bolinha "pula-pula" e um transferidor de papel. O pêndulo montado pode ser visto na Fig. 2a.
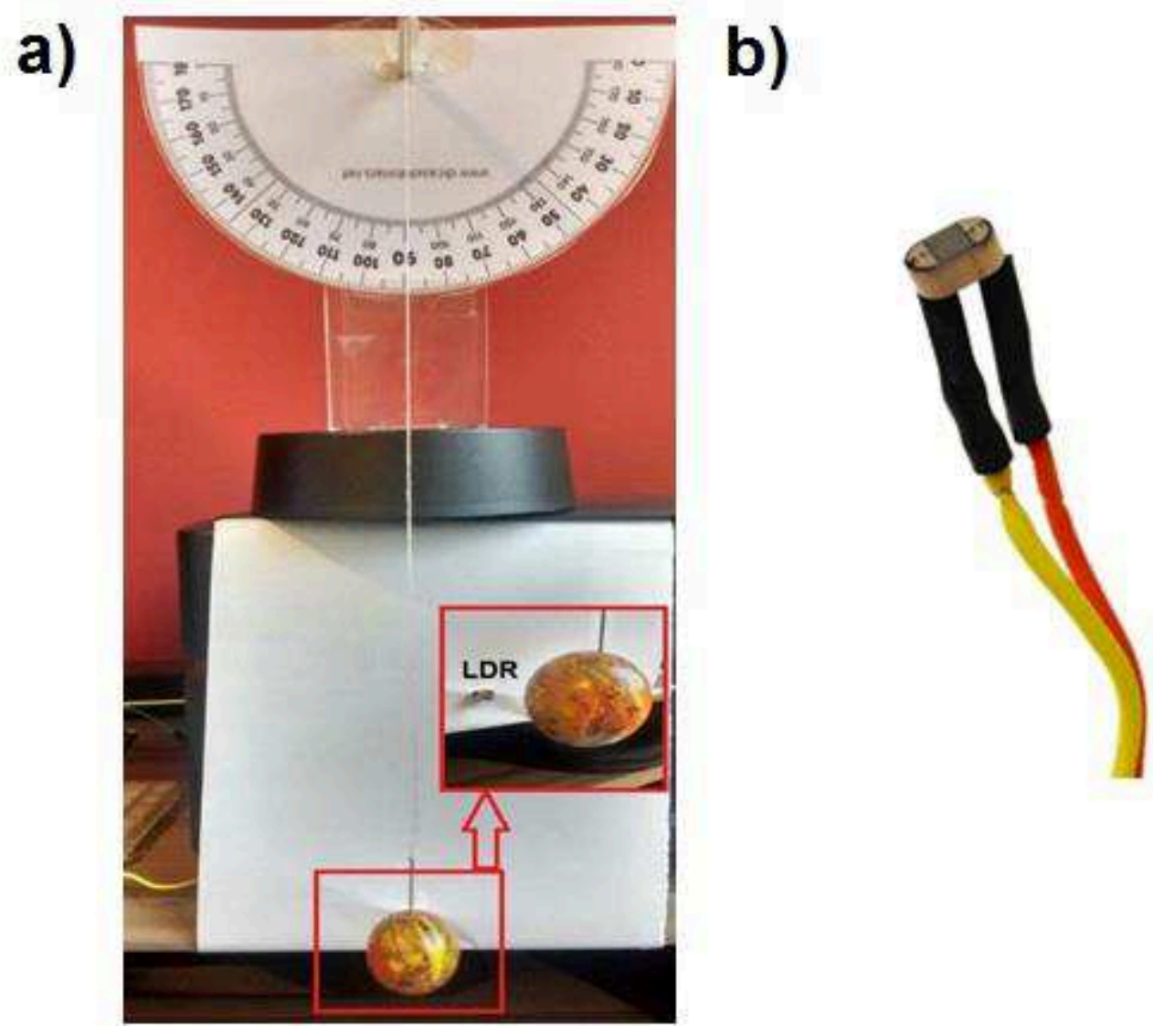

Fig. 2 - Montagem do pêndulo à esquerda (a) e sensor LDR à direita (b).

\section{III.2 LDR e circuito utilizado}

O LDR é um resistor com resistência variável dependente da luz incidente, desta maneira pode ser utilizado para medir indiretamente a luminosidade de um ambiente em escala arbitrária ou em lux, caso seja feita a conversão adequada. É de baixíssimo custo e pode ser facilmente adquirido. Seu funcionamento ocorre da seguinte forma: constrói-se um circuito com o $L D R$ e um capacitor em série, ou seja, um circuito $\mathrm{RC}$, e então liga-se o circuito à placa Raspberry Pi. Realizam-se então continuamente ciclos onde o capacitor é totalmente descarregado e carregado até que a voltagem percorrida seja de 1,4 V - voltagem onde o pino digital de entrada do Raspberry Pi "compreende" que o estado do sinal enviado é "HIGH" -, cada ciclo com duração que varia entre 10 e 200ms - constante de tempo do circuito, obtida pelo produto da resistência do LDR com a capacitância do capacitor. Durante o carregamento o programa cronometra o tempo de carga $\tau_{n}$, que por ser diretamente proporcional ao produto da resistência 
pela capacitância é uma medida indireta da resistência momentânea do $L D R$ e também da luminosidade sobre ele. $\mathrm{O}$ código utilizado imprime então num arquivo os valores $\tau_{n}$ e o correspondente tempo da medição, $t_{n}$. Em nosso arranjo é colocado diretamente sobre o $L D R$ um feixe direto de luz (foi utilizada uma lanterna de smartphone) e entre o feixe e o LDR está a posição de mínima altura do pêndulo, associada a $\theta=0$. Cada vez que o pêndulo passar por esta posição o $L D R$ ficará “escuro", e teremos um ponto de máximo em $\tau_{n}$, enquanto nos outros momentos o $L D R$ ficará “claro", com um valor menor para $\tau_{n}$. O período do pêndulo $T_{n}$ será o tempo transcorrido entre 3 máximos consecutivos, ou seja, $T_{n}=t_{M, n+1}-t_{M, n-1}$. O capacitor utilizado no experimento é um capacitor cerâmico com capacitância de $1 \mu F$, e a resistência do $L D R$ utilizado varia de aproximadamente $10 k \Omega$ no "claro" a $200 k \Omega$ no "escuro". Como o $\tau_{n}$ é diretamente proporcional à resistência do circuito $\mathrm{RC}$, a medição desta grandeza será cerca de 10x maior no "escuro" do que no "claro". É importante notar que a resistência do LDR nunca chegará a $200 \mathrm{k} \Omega$, visto que este valor está associado ao $L D R$ numa situação de total ausência de luz. Uma análise detalhada do circuito RC pode ser encontrada em diversos livros didáticos (NUSSENZVEIG, 2002). Devido à grande comunidade de usuários do Raspberry Pi é possível obter códigos prontos com facilidade nos fóruns de discussão da placa em grupos do facebook (como o grupo Raspberry Pi Brasil) e em algumas páginas (ROBOZERA, 2017; RASPBERRY PI FOUNDATION, 2017; PI MY LIFE UP, 2017).

O circuito utilizado é bastante simples, e pode ser visto na Figura 1 e esquematicamente na Fig. 3. Uma ponta do $L D R$ é ligada ao pino de 3,3 V da Raspberry Pi, e a outra ponta a um fio que leva a um pino de leitura da placa, no caso o GPIO18. Neste mesmo GPIO18 é ligado o positivo do capacitor, enquanto o negativo é ligado ao GROUND da placa. Caso numa reprodução seja utilizado outro pino diferente do GPIO18 é necessário modificar o código presente no apêndice A.

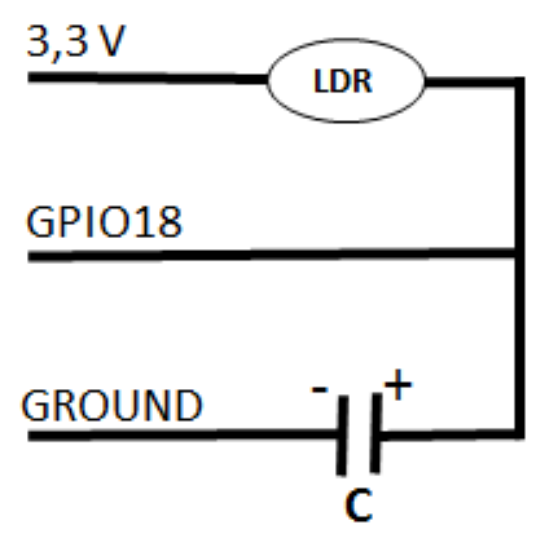

Fig. 3 - Esquema do circuito utilizado. 


\section{III.3 Configurando a Placa}

Para utilizar o Raspberry Pi o primeiro passo é ir na página oficial da fabricante (raspberrypi.org) e na seção downloads baixar o NOOBS. Após ser baixado, descompacte os arquivos e transfira-os para um cartão micro SD de no mínimo 8GB previamente formatado. Caso seu computador não tenha um leitor de cartões SD utilize um adaptador USB. Feito isso, introduza o cartão no Raspberry Pi, conecte mouse, teclado e um monitor e ligue-o na tomada. Salientamos que este pequeno tutorial foi escrito utilizando a versão 2.4.0 do NOOBS, e pode sofrer alterações para versões posteriores.

Ao ligar aparecerá uma tela de instalação, selecione "Raspbian with PIXEL" e aperte "install", o processo dura entre 10 e 15 minutos, e após o término aperte "ok" e aguarde a inicialização do sistema. Iniciado o sistema, a primeira coisa a se fazer é conectar à internet para instalar alguns pacotes necessários, então plugue um cabo de rede diretamente na placa ou acesse uma rede wifi através do segundo ícone no canto superior direito. Em seguida abra o Terminal clicando no quarto ícone no canto superior esquerdo. Digite os seguintes comandos, sudo apt-get update (tecle enter e aguarde)

sudo apt-get install python-dev (aperte enter e aguarde, digite y quando necessário) sudo apt-get install python-rpi.gpio (aperte enter e aguarde)

Agora, digite este comando para criar o arquivo onde colocaremos nosso programa, sudo nano LDR.py (tecle enter)

Escreva na tela que abrirá o código que está no apêndice A, ao fim aperte os botões ctrl e x juntamente para fechar, aperte y para salvar e aperte enter. Um caminho mais fácil é copiar diretamente para seu Raspberry Pi o programa através de nosso github utilizando o comando,

git clone https://github.com/thiagoca3/LDR/(tecle enter e espere)

cd./LDR (tecle enter)

Agora, com o programa em seu Raspberry Pi e o circuito corretamente montado, dê o comando

sudo python LDR.py (tecle enter)

O programa então começará a rodar e mostrar os valores do LDR na tela seguido pelo tempo correspondente. Faça testes deixando o LDR em luz e sombra e verá que o valor mostrado na tela varia. Para encerrar o programa aperte juntos os botões ctrl e c. Todos os dados (leitura do LDR e tempo da medição) estarão sendo salvos no arquivo results.txt que estará no desktop, caso tenha criado o arquivo, ou na pasta LDR, caso tenha baixado de nosso github. Clique no terceiro ícone superior do lado esquerdo para abrir o gerenciador de arquivos e visualizar seus resultados no arquivo. 


\section{Resultados experimentais}

Manipulando a Eq. (2) podemos obter facilmente uma expressão para a aceleração da gravidade, dada por

$$
g=\frac{L}{\bar{T}^{2}}\left(\frac{2 \pi}{\operatorname{agm}(1, \cos (\theta / 2))}\right)^{2} .
$$

Na experiência realizada, utilizamos $L=0,293 \mathrm{~m}$, e consideramos apenas as cinco primeiras medidas de período realizadas, calculando o período médio $\bar{T}$, já que as seguintes não corresponderiam mais ao ângulo inicial. Realizamos medidas para os ângulos $\theta=10^{\circ}, 20^{\circ}$, $30^{\circ}, 40^{\circ}$ e $50^{\circ}$. Uma discussão acerca das incertezas pode ser encontrada no apêndice B.

Na Tabela 1 temos os valores obtidos de $\bar{T}$ e $g$ para cada ângulo, assim como suas respectivas incertezas.

Tabela 1: Valor médio do período $\mathrm{T}$ obtido considerando as 5 primeiras medições em cada ângulo e a aceleração da gravidade calculada.

\begin{tabular}{|r|l|l|}
\hline$\theta$ (graus) & $\bar{T}(s)$ & $g\left(\mathrm{~m} / \mathrm{s}^{2}\right)$ \\
\hline $10^{\circ}$ & $1,09 \pm 0,01$ & $9,77 \pm 0,05$ \\
\hline $20^{\circ}$ & $1,10 \pm 0,01$ & $9,71 \pm 0,05$ \\
\hline $30^{\circ}$ & $1,10 \pm 0,01$ & $9,89 \pm 0,06$ \\
\hline $40^{\circ}$ & $1,12 \pm 0,01$ & $9,81 \pm 0,06$ \\
\hline $50^{\circ}$ & $1,15 \pm 0,01$ & $8,75 \pm 0,07$ \\
\hline
\end{tabular}

Podemos notar que o valor obtido para $g$ em $50^{\circ}$ está bem abaixo dos valores obtidos em outros ângulos. Devido à simplicidade do aparato experimental, oscilações nesta faixa acabaram por causar um mal funcionamento, com oscilações muito irregulares. No entanto, o valor do período não está muito além do valor esperado para o mesmo utilizando um $g$ obtido na literatura (SOUSA, 2010) para a cidade do Rio de Janeiro $\left(T_{t e o, 50^{\circ}}=1,141 \mathrm{~s}\right)$. Se tomarmos a média de nossos quatro melhores valores de $g$ teremos o seguinte $g_{\text {exp }}$ obtido pelo experimento,

$$
g_{\text {exp }}=9,79 \pm 0,03 \mathrm{~m} / \mathrm{s}^{2},
$$

e se compararmos com o $g$ obtido na literatura, com as devidas aproximações e conversões para efeito de comparação, teremos o seguinte desvio percentual relativo,

$$
\frac{\left|\bar{g}-g_{l i t}\right|}{\bar{g}}=\frac{|9,795-9,788|}{9,795} \approx 0,07 \% \text {, }
$$

que representa um excelente acordo para um experimento realizado com tamanha simplicidade. 


\section{Conclusões}

Apresentamos a placa Raspberry Pi como uma opção de baixo custo para a elaboração de um laboratório portátil. Para comprovar a aplicabilidade realizamos a análise do pêndulo simples obtendo o valor da aceleração da gravidade em excelente acordo com valor encontrado na literatura. O material utilizado é de baixíssimo custo, somando não mais que $\mathrm{R} \$ 10$. A placa e os acessórios necessários para sua utilização (fonte, cartão SD e case) podem ser adquiridos em páginas de importação, a um valor não superior a $\mathrm{R} \$ 200$. Desta forma, com um gasto de no máximo $\mathrm{R} \$ 300$, é possível obter a placa, acessórios e uma gama enorme de sensores, elementos de circuito e outros dispositivos que possibilitam a realização de uma diversidade de experimentos relacionados às diversas áreas da Física. Para importação recomendamos as lojas $G e$ arbest, modmypi, banggood e aliexpress - o tempo de importação varia de 30 a 45 dias e o produto pode ser taxado, em valores que variam de 30 a 50 reais. Caso prefira comprar o produto já no Brasil ele está à venda em algumas lojas virtuais de eletrônicos. O melhor preço pode ser encontrado em vendedores do Mercado Livre, onde apenas a placa tem custo médio de $\mathrm{R} \$ 200$, e com acessórios pode chegar a cerca de $\mathrm{R} \$ 300$.

As possibilidades de aprendizado não se resumem somente às experiências em si, mas também no estudo dos sensores e na montagem do circuito. No experimento apresentado é possível abordar não só o estudo do oscilador harmônico através do pêndulo, como também o estudo de circuitos, através da montagem do circuito utilizado e também do funcionamento do $L D R$. O mesmo experimento pode ainda ser realizado de diversas maneiras, como por exemplo utilizando um sensor ultrassônico para detectar a passagem da massa do pêndulo ou um sensor de infravermelho, permitindo ao professor realizar um estudo com os alunos dos prós e contras de cada maneira de realizar o experimento e da limitação de cada uma. Também é possível realizar a medição de $g$ por outros métodos, como por exemplo pelo estudo da queda de um corpo, e da mesma forma o professor pode analisar com os alunos as vantagens e desvantagens dos diferentes métodos. $\mathrm{O}$ Raspberry $P i$ apresenta uma vantagem de custo em relação à utilização do Arduíno pelo fato deste necessitar adicionalmente de um computador, enquanto que o Raspberry Pi já é um computador, necessitando apenas de um teclado e um monitor para utilização.

Almejamos desenvolver material didático completo para que interessados em geral possam utilizar o Raspberry Pi como ferramenta de ensino, sem necessidade de conhecimento prévio acerca de programação ou montagem de circuitos. Também pretendemos promover cursos de extensão no Instituto de Aplicação Fernando Rodrigues da Silveira (CAp/UERJ) para treinamento do público em geral na utilização da placa.

\section{Agradecimentos}

Agradecemos à FAPERJ pelo auxílio fornecido e à Prof. ${ }^{a}$ Manoela Lopes Carvalho pelas sugestões dadas. 


\section{Referências Bibliográficas}

AGGARWAL, N.; VERMA, N.; ARUN, P. Simple pendulum revisited. European Journal of Physics, v. 26, p. 517, 2005.

CARVALHAES, C. G.; SUPPES, P. Approximations for the period of the simple pendulum based on the arithmetic-geometric mean. American Journal of Physics, v. 76, p. 1150, 2008.

CAVAlCANTE, M. A.; TAVOLARO, C. R. C.; MOLISANI, E. Física com arduino para iniciantes. Revista Brasileira de Ensino de Física, São Paulo, v. 33, n. 4, p. 4503, out. 2011.

CHANDRAPPA, S. et al. Design and Development of IoT Device to Measure Quality of Water. International Journal of Modern Education and Computer Science, Hong Kong, v. 9, n. 4, p. 50-56, Apr. 2017.

CORDOVA, H.; TORT, A.C. Medida de g com a placa arduino em um experimento simples de queda livre. Revista Brasileira de Ensino de Física, São Paulo, v. 38, n. 2, p. 2308, maio 2016.

FAGUNDES, D. et al. Usando a porta paralela do micro PC. Revista Brasileira de Ensino de Física, São Paulo, v. 17, n. 2, p. 196-201, jun. 1995.

HALLIDAY, D; RESNICK, R.; WALKER, J. Fundamentos de Física. 8. Ed. Rio de Janeiro: Livros Técnicos e Científicos, 2009. v. 2.

MAINA, C.; MUHIA, A.; OPONDO, J. A Low Cost Laboratory for Enhanced Electrical Engineering Education. 2016 IST-Africa Week Conference, Durban, p. 1-8, 2016.

MAULANA, F.; SOEGIJOKO, W.; YAMANI, A. Utilising Raspberry Pi as a cheap and easy do it yourself streaming device for astronomy. Journal of Physics, v. 771, n. 1, 012025, 2016.

MENEZES, C.; NEY, N. Introdução à Programação Com Python. São Paulo: Novatec, 2014.

NATARAJAN, S. et al. Low Cost Temperature Logging System using Raspberry Pi. International Research Journal of Engineering and Technology, Trichy, v. 4, n. 4, p. 254-258, Apr. 2017.

NUSSEnZVEIG, H. M. Curso de Física Básica. 5. ed. São Paulo: Blucher, 2013. v. 2.

PI MY LIFE UP. Pi My Life Up. Disponível em: <https://pimylifeup.com>. Acesso em: 22 Abr. 2017.

RASPBERRY PI FOUNDATION. Raspberry Pi Foundation. Disponível em: <https://www.raspberrypi.org/>. Acesso em: 22 Abr. 2017. 
ROBOZERA. ROBOZERA. Disponível em: <https://robozera.com>. Acesso em: 22 Abr. 2017.

SOUSA, D. F. et al. Aquisição de dados e aplicações simples usando a porta paralela do micro PC. Revista Brasileira de Ensino de Física, São Paulo, v. 20, n. 4, p. 413-422, dez. 1998.

SOUSA, M. A.; SANTOS, A. A. Absolute gravimetry on the Agulhas Negras calibration line. Rev. Bras. Geof., Rio de Janeiro, v. 28, n. 2, p. 165, abril 2010.

SOUZA, A. R. et al. A placa arduino: uma opção de baixo custo para experiências de física assistidas pelo PC. Revista Brasileira de Ensino de Física, São Paulo, v. 33, n. 1, p. 1702, jan. 2011.

UPTON, E.; HALFACREE, G. Raspberry Pi: Manual do Usuário. São Paulo: Novatec, 2013. VUOLO, J. H. Fundamentos da Teoria de Erros. 2. Ed. São Paulo: Blucher, 1996. 


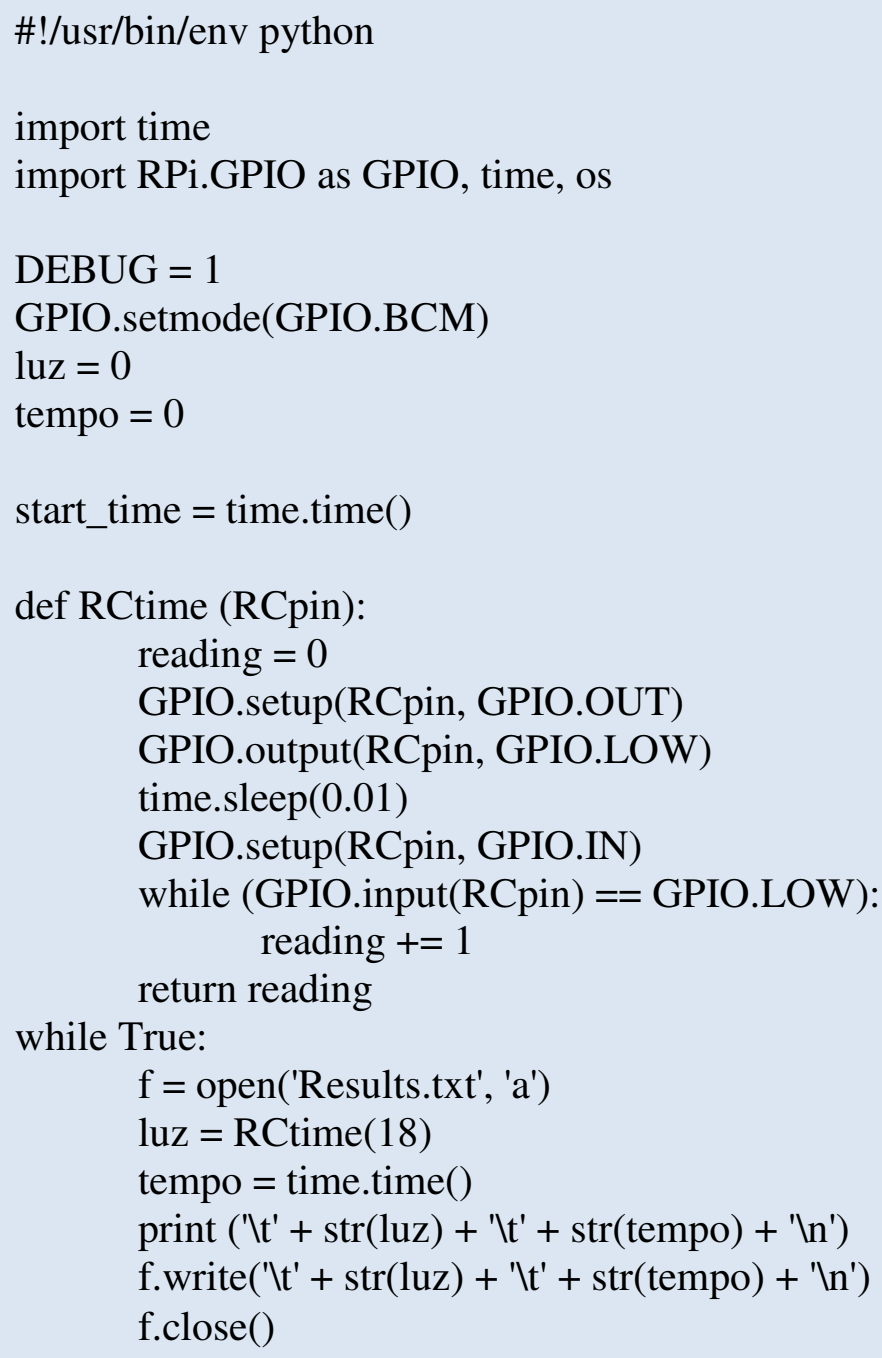

\section{Apêndice B: incerteza padrão em $g$}

Segundo Vuolo (1996), dada uma grandeza $w(x, y, z, \ldots)$, onde as grandezas experimentais $x, y, z, \ldots$ possuem incertezas padrões independentes entre si $\sigma_{x}, \sigma_{y}, \sigma_{z}, \ldots$ respectivamente, a incerteza padrão em $w$ será dada por,

$$
\sigma_{w}^{2}=\left(\frac{\partial w}{\partial x}\right)^{2} \sigma_{x}^{2}+\left(\frac{\partial w}{\partial y}\right)^{2} \sigma_{y}^{2}+\left(\frac{\partial w}{\partial z}\right)^{2} \sigma_{z}^{2}+\cdots
$$

Sendo assim, para a aceleração da gravidade obtida através da Eq. (3) temos a seguinte incerteza padrão,

$$
\sigma_{g}^{2}=\left(\frac{\partial g}{\partial L}\right)^{2} \sigma_{L}^{2}+\left(\frac{\partial g}{\partial \bar{T}}\right)^{2} \sigma_{\bar{T}}^{2}+\left(\frac{\partial g}{\partial \theta}\right)^{2} \sigma_{\theta}^{2}
$$


Realizando as derivadas parciais temos então,

$$
\sigma_{g}^{2}=k^{4}\left[\left(\frac{1}{\bar{T}^{2}}\right)^{2} \sigma_{L}^{2}+\left(\frac{2 L}{\bar{T}^{3}}\right)^{2} \sigma_{\bar{T}}^{2}+\left(\frac{L \sin (\theta / 2)}{\bar{T}^{2} \operatorname{agm}(1, \cos (\theta / 2))}\right)^{2} \sigma_{\theta}^{2}\right]
$$

sendo

$$
k=\frac{2 \pi}{\operatorname{agm}(1, \cos (\theta / 2))}
$$

Os valores das incertezas padrão do comprimento do pêndulo $(L)$, do período médio computado $(\bar{T})$ e do ângulo $(\theta)$, são respectivamente:

$$
\sigma_{L}=0,001 \mathrm{~m}, \sigma_{\bar{T}}=\sigma_{T} /_{\sqrt{n}}=0,002 \mathrm{~s} \text { e } \sigma_{\theta}=0,009 \mathrm{rad} .
$$

A incerteza padrão para o período é a metade do tempo de cada ciclo realizado pelo programa, conforme discutido na seção III.2, e estamos utilizando um período médio dos cinco primeiros períodos obtidos $(n=5)$. 